\title{
Application of spectral analysis in radiometric measurements of two- phase liquid-gas flow
}

\author{
Marcin Zych ${ }^{1, a}$, Robert Hanus ${ }^{2}$ and Leszek Petryka ${ }^{1}$ \\ ${ }^{1}$ AGH University of Science and Technology, Krakow 30-059, Poland \\ ${ }^{2}$ Rzeszow University of Technology, Rzeszow 35-959, Poland
}

\begin{abstract}
This article presents use of the classical spectral analysis to identify a type of flow in investigation of gas transportation by liquid with a measurement of gamma radiation absorption. During numerous experiments it was found that a magnitude of the cross-spectral density distribution of recording signals reveals type of air-water mixture flow in a horizontal pipeline. As an example, some results of laboratory measurements equipped in ${ }^{241} \mathrm{Am}$ radiation source and scintillation probes with of $\mathrm{NaI}(\mathrm{Tl})$ crystals are presented. Moreover attached figures facilitate interpretation of observed results and in details illustrating the proposed method.
\end{abstract}

\section{Introduction}

The flow when gas is transporting by liquid occurs in many industries, especially in heat transfer installations. Depending of the liquid velocity, viscosity, and presence of micro turbulences, the same volume of gas may be transported in a different way, evolving from slug, up to homogenous or annular flow. Hence there is a need for development of methods of continuous observation and mathematical description of such type of flow.

Currently, those flows may be examined by such methods as computer tomography (resistive, capacitive, optical and X-rays), Coriolis flowmeters, optical equipment, and nuclear techniques [1-6]. The latter one are also used by the authors of this article in studies of gas-liquid and liquid-solids flows [7-10].

In our case the nuclear method base on application of radiotracers and (or) using a gamma radiation for the flow analysis. The last of those methods is especially convenient for continuous observation of unstable twophase flows, providing velocity of gas phase and manner of its transportation. That goal is achieved by advanced analysis of signals delivered by scintillation probes in time and frequency domains.

Flow regime identification inspires many studies of two-phase flows. Recent works shows use for this purpose the power spectral density, wavelet transform, Hilbert-Huang transform, artificial neural networks and expert systems, electrical capacitance tomography and fuzzy-logic classification [11-15].

The article provided use of the cross-spectral density function (CSDF) to identify the type of flow, illustrated by the attached examples.

\footnotetext{
a Corresponding author: zych@geol.agh.edu.pl
}

\section{Laboratory stand}

The paper, based on results collected in the pilot installation built at the Laboratory of Industrial Radiometry, Faculty of Physics and Applied Computer Science, AGH in Krakow [16]. The simplistic schema of the stand is shown in Figure 1.

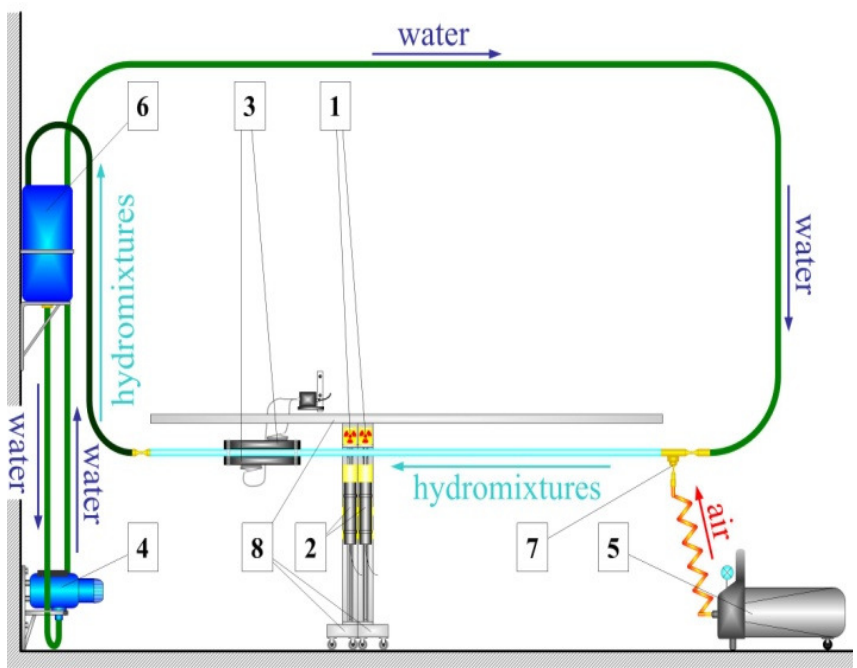

Figure 1. Scheme of experimental hydraulic installation: 1 - $\gamma$-ray sealed source, 2 - scintillation probe, 3 - ultrasound flow meter, 4 - pump, 5 - air compressor, 6 - air-removing vessel, 7 - air nozzle, 8 - shifting system.

The installation consists of a transparent acrylic glass tube with internal diameter $30 \mathrm{~mm}$ and a length of $4.5 \mathrm{~m}$, 
to which water is pumped by a rotary pump (4) and air from the compressor (5) by an injector nozzle (7). Due to that the controlled mixture of water and air supply the measuring pipe and flowing up to the air-removing tank (6). The measurement of air bubbles velocity and flow regime recognition was arrange by the measuring system consisting of two linear $\gamma$-radioactive sources, ${ }^{241} \mathrm{Am}$ (1) with an activity of $100 \mathrm{mCi}$ and two probes with $\mathrm{NaI}(\mathrm{Tl})$ (2) scintillation detector. The measuring set is mounted on a special trolley (8), which allows moving the set along the pipeline. Water flowrate was continuously measured by Uniflow 990 ultrasonic flowmeter (3). The rotary pump (4) controlled by a waver allows adjustment of the revolution in range between 1000 and $2800 \mathrm{rpm}$, which allows selection of water velocity in the measuring pipe between $0.5-2.5 \mathrm{~m} / \mathrm{s}$.

The data acquisition system was connected with a PC by the dedicated counters card and USB port. The general view of the stand during nuclear measurement is shown in Figure 2.

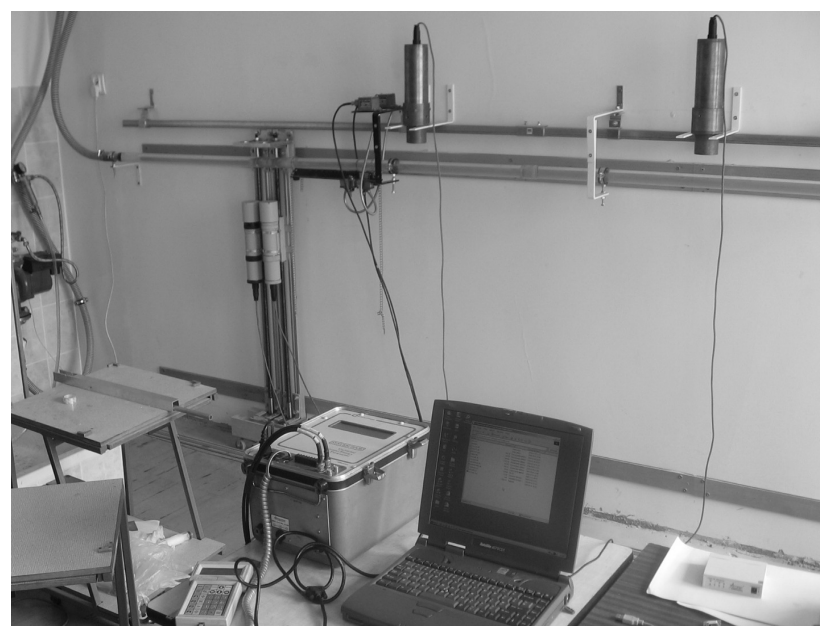

Figure 2. General view of the measuring stand during tracer and absorption experiment

Transparent measuring section of the pipeline allows additionally a photographic documentation of the observed phenomena.

\section{Application of Gamma absorption in two-phase flow measurement}

The idea and geometry of the measurement are shown in figure 3. Two ${ }^{241} \mathrm{Am}$ sealed radioactive sources (2) emit $\gamma$ radiation beams $(6,7)$, shaped by the collimator (1). Photons pass through the pipeline with analyzed mixture (5) and collimator (3) before achieved the detector (4).

In investigations the linear radiation sources ${ }^{241} \mathrm{Am}, \mathrm{QSA}$ Global were used, which structure and dimensions are shown in figure 4.

The data acquisition equipment allows recording of $I_{x}(t)$ and $I_{y}(t)$ intensity representing the composition of the flowing mixture. Results recorded in the BUB006 experiment are shown in Figure 5.

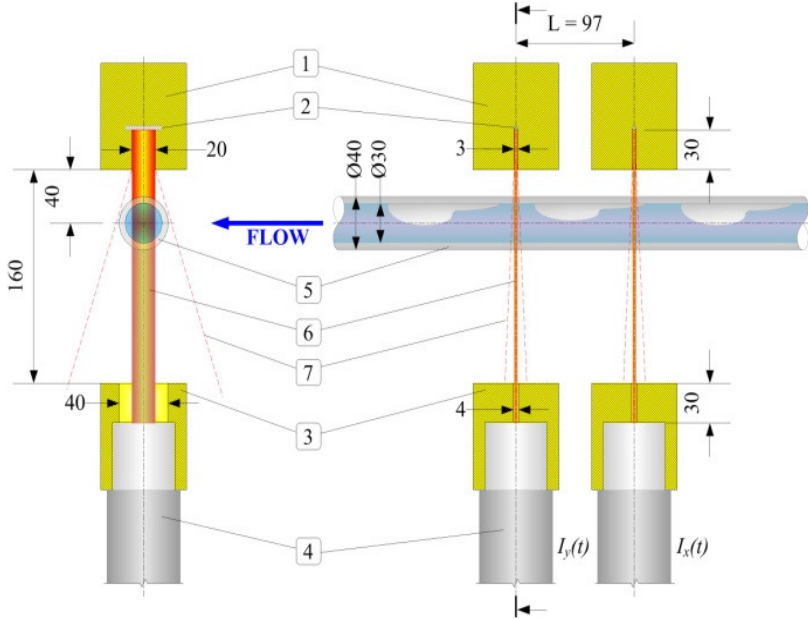

Figure 3. The principle geometry of $\gamma$-ray absorption measurement: 1 - collimator, $2-{ }^{241} \mathrm{Am}$ source, 3 - collimator of detector 4 - scintillation probe, 5 - pipeline, 6 - main $\gamma$-ray beam, 7 - external $\gamma$ radiation. All dimensions are given in $\mathrm{mm}$.

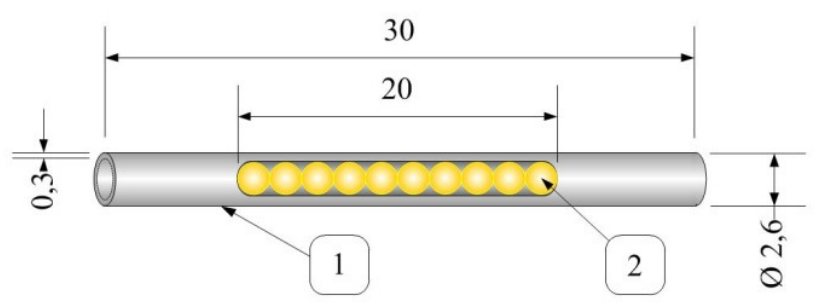

Figure 4. Scheme of $\gamma$ source ${ }^{241} \mathrm{Am}$, QSA Global: 1 - stainless steel, 2 - radioactive material. All dimensions are given in $\mathrm{mm}$.

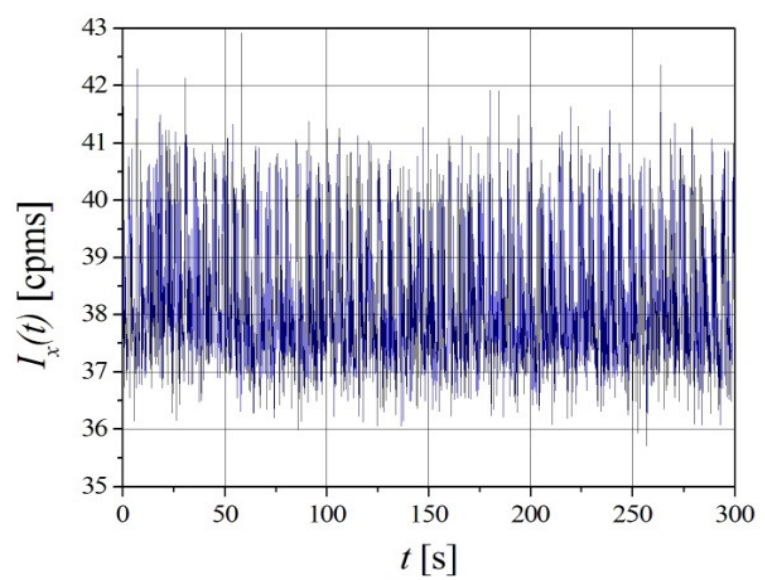

Figure 5. Intensity of $\gamma$-ray beam recorded by the first probe in the run BUB006

\section{The spectral analysis of recorded signals}

The TTL stochastic pulses $I_{x}(t)$ and $I_{y}(t)$ counted during the sample period $\Delta t=1 \mathrm{~ms}$ form discrete measuring signals $x(n)$ and $y(n)$. These signals after Fourier Transform are used for analysis in the frequency domain.

In the paper the autospectral density function $(A S D F)$ and cross-spectral density function $C S D F$, determined unilateral in practice (for the frequency range $0<f<\infty$ ) $A S D F: G_{x x}(f), G_{y y}(f)$ and $C S D F: G_{x y}(f)$ can be expressed by the following formulas [17]: 


$$
\begin{aligned}
& G_{x x}(f)=2 \int_{-\infty}^{\infty} R_{x x}(\tau) e^{-j 2 \pi f \tau} d \tau \\
& G_{y y}(f)=2 \int_{-\infty}^{\infty} R_{y y}(\tau) e^{-j 2 \pi f \tau} d \tau \\
& G_{x y}(f)=2 \int_{-\infty}^{\infty} R_{x y}(\tau) e^{-j 2 \pi f \tau} d \tau
\end{aligned}
$$

where: $R_{x x}(\tau), R_{y y}(\tau)$ and $R_{x y}(\tau)$ are the autocorrelation and cross-correlation function properly, $f$ - frequency, $\tau$ - time delay.

During data processing the Fast Fourier Transform $(F F T)$ was used directly to the discrete $x(n)$ and $y(n)$ signals. In consequence the phase distribution of CSDF were used to measure the average velocity of air bubbles, as the minority phase of the mixture $[8,18]$. The same results were obtained by the cross-correlation analysis facilitated by the band pass filtering of the recorded signals $[16,19]$.

Additionally the spectral analysis can be used to recognize the type of the gas bubbles transportation in the flow. The literature shows a use for this purpose $A S D F$ [10], however, according to the authors, better results can be accomplished using a magnitude of cross-spectral density $|C S D F|$. For illustration the figure 6 shows $A S D F$ and $|C S D F|$ designated for data recorded in the experiment BUB006 (slug flow of water -air mixture).

a)

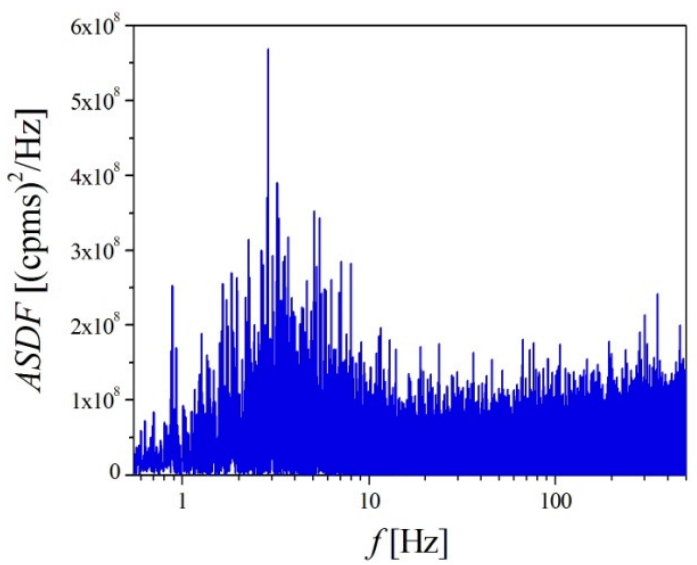

b)

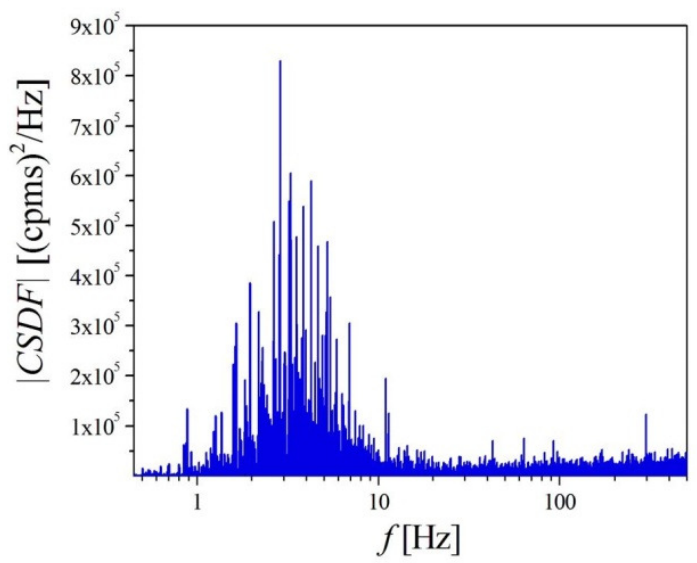

Figure 6. Comparison of the $A S D F$ (a) and $|C S D F|$ (b) calculated in the run BUB006.

The comparison of figure $6 \mathrm{a}$ and $6 \mathrm{~b}$ shows that the frequency range associated with the used signal is more apparent in the course of $|C S D F|$ than $A S D F$.

\section{Application of CSDF to identify the type of flow}

During experiments with air volume concentration in the flow equal 0.3 , increasing water velocity $v_{W}$ gives the following forms of flow:

- $v_{W}=0.5 \div 1.0 \mathrm{~m} / \mathrm{s}-$ plug flow $\quad$ BUB006,

- $v_{W}=1.0 \div 1.6 \mathrm{~m} / \mathrm{s}-$ slug flow $\quad$ BUB004,

- $v_{W}=1.6 \div 2.5 \mathrm{~m} / \mathrm{s}-$ bubble flow BUB001.

The results of processing of data recorded in experiments BUB001, BUB004 and BUB006 are presented in Figure 7. Such arrange facilities observation of flow evolution as well as its influence on the CSDF distributions. In consequence it is easy to observe how changing flow velocity results in variation of the form of transportation the same volume of gas and modifying absorption of gamma radiation. Moreover the figure illustrates advantages of the CSDF distributions application to recognition the state of the flowing mixture.

a)
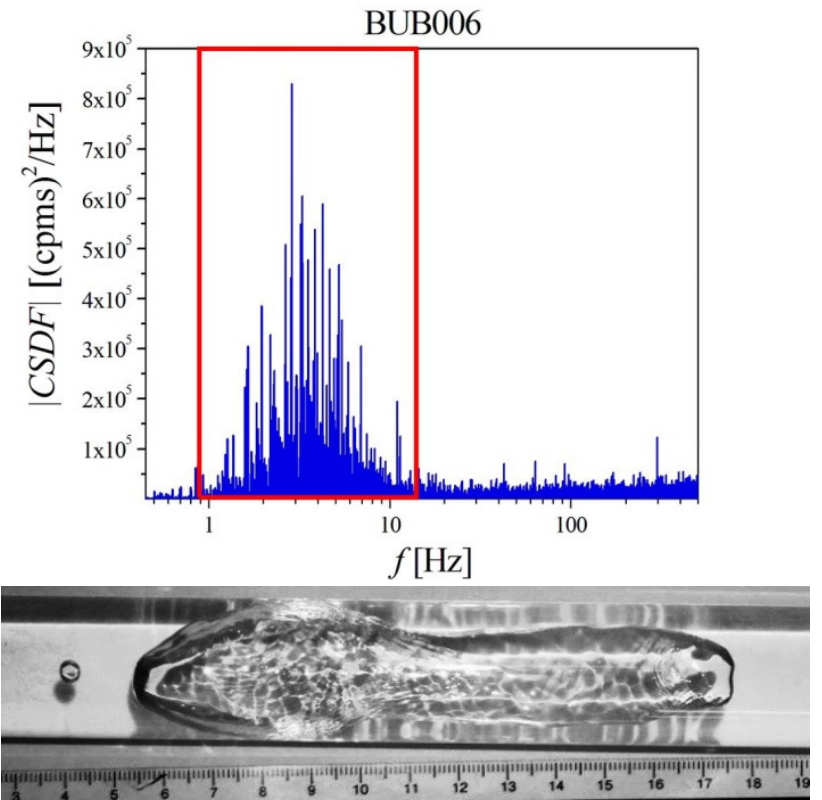
b)
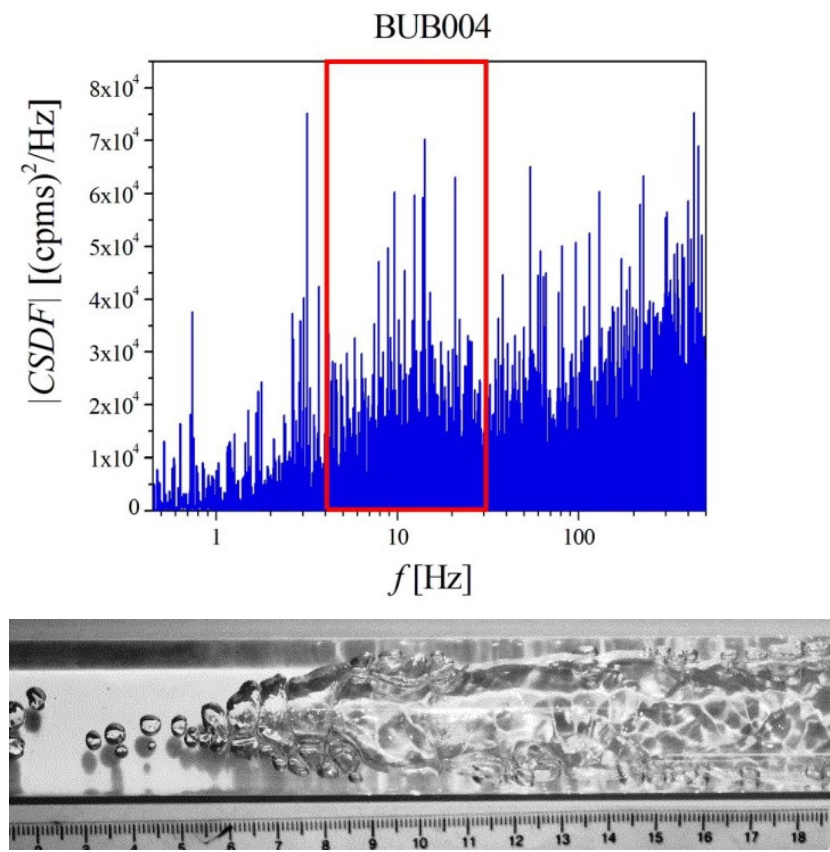

c)
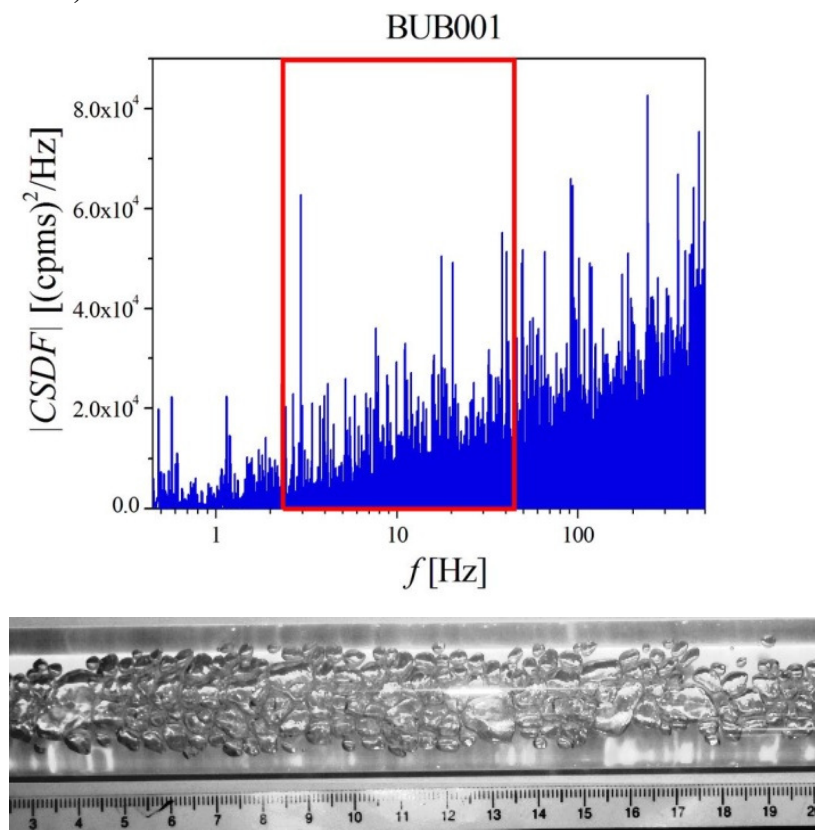

Figure 7. Cross-spectral density of recorded signals during selected kind of the flow: a) run BUB006 - plug flow, $v_{\mathrm{W}}=0,90 \mathrm{~m} / \mathrm{s}, v_{\mathrm{A}}=0,710 \mathrm{~m} / \mathrm{s} ;$ b) run BUB004 - slug flow, $v_{\mathrm{W}}=1,36 \mathrm{~m} / \mathrm{s}, \quad v_{\mathrm{A}}=1,066 \mathrm{~m} / \mathrm{s} ; \mathrm{c}$ ) run BUB001 - bubble flow, $v_{\mathrm{W}}=1,92 \mathrm{~m} / \mathrm{s}, v_{\mathrm{A}}=1,446 \mathrm{~m} / \mathrm{s}$. Red frame marked the most representative spectrum.

\section{Conclusion}

Competed investigations justified advantages of the gamma absorption techniques application to examination of two phase flow and simultaneous determination both the velocity of gas bubbles and pattern recognition of its forms. Moreover collected results prove advantages of cross-spectral density in compare to autospectral density function to processing recorded signals.

Due to that the proposed method is worthwhile to consider application in other laboratories or even to control of selected heating installations.

\section{References}

1. Ting Xue, Liqun Qu, Zhaofeng Cao, Tao Zhang, Three-dimensional feature parameters measurement of bubbles in gas-liquid two-phase flow based on virtual stereo vision. Flow Measurement and Instrumentation 27, 29-36 (2012)

2. M. Bottin, J.P. Berlandis, E. Hervieu, M. Lance, M. Marchand, O.C. Öztürk, G. Serre, Experimental investigation of a developing two-phase bubbly flow in horizontal pipe. International Journal of Multiphase Flow 60, 161-179 (2014)

3. G. Falcone, G.F. Hewitt, C. Alimonti, Multiphase flow metering: principles and applications. (Elsevier Amsterdam, 2009)

4.

M.R. Rzasa, The measuring method for tests of horizontal twophase gas-liquid flows, using optical and capacitance tomography. Nuclear Engineering and Design 239, 699-707 (2009)

5. V. Mosorov, A method of transit time measurement using twin plane electrical tomography. Meas. Science and Technology 17, 753-760 (2006)

6. S. Blaney, H. Yeung, Investigation of the exploitation of a fast-sampling gamma densitometer and pattern recognition to resolve the superficial phase velocities and liquid phase water cut of vertically upward multiphase flows. Flow Measurement and Instrumentation 19, 57-66 (2007)

7. M. Zych, L. Petryka, J. Kępiński, R. Hanus, T. Bujak, E. Puskarczyk, Radioisotope investigations of compound two-phase flows in an open channel, Flow Measurement and Instrumentation 35, 11-15 (2014)

8. L. Petryka, R. Hanus, M. Zych, M. Śleziak, Radioisotope measurements of two-phase flow. Przegląd Elektrotechniczny 86, 5, 24-29 (2010) (in Polish)

9. R. Hanus, A. Szlachta, A. Kowalczyk, L. Petryka, M. Zych, Radioisotope Measurement of Two-Phase Flow in Pipeline Using Conditional Averaging of Signal. Proc. 2012 IEEE Mediterranean Electrotechnical Conference MELECON 2012, 
Medina Yasmine-Hammamet, Tunisia, March 2528, 144-147 (2012)

10. L. Petryka, M. Zych, R. Murzyn, The non-stationary two-phase flow evaluation by radioisotopes. Nukleonika 50, 43-46 (2005)

11. L. Liebenberg, J.P. Meyer, The characterization of flow regimes with power spectral density distributions of pressure fluctuations during condensation in smooth and micro-fin tubes. Experimental Thermal and Fluid Science 31, 127140 (2006)

12. Hao Ding, Zhiyao Huang, Zhihuan Song, Yong Yan, Hilbert-Huang transform based signal analysis for the characterization of gas-liquid two-phase flow. Flow Measurement and Instrumentation 18, 37-46 (2007)

13. T. Elperin, M. Klochko, Flow regime identification in a two-phase flow using wavelet transform. Experiments in Fluids 32, 674-682 (2002)

14. E.S. Rosa, R.M. Salgado, T. Ohishi, N. Mastelari, Performance comparison of artificial neural networks and expert systems applied to flow pattern identification in vertical ascendant gas-liquid flows. International Journal of Multiphase Flow 36, 738754 (2010)

15. R. Banasiak, R. Wajman, T. Jaworski, P. Fiderek, H. Fidos, J. Nowakowski, D. Sankowski, Study on two-phase flow regime visualization and identification using 3D electrical capacitance tomography and fuzzy-logic classification. International Journal of Multiphase Flow 58, 1-14 (2014)

16. L. Petryka, R. Hanus, M. Zych, Application of gamma absorption method to two phase flow measurement in pipelines. Przeglad Elektrotechniczny 88, 1b, 185-188 (2012) (in Polish).

17. J.S. Bendat, A.G. Piersol, Random data - analysis and measurement procedures. (John Wiley, New York, 2000)

18. R. Hanus, Statistical error analysis of time delay measurement by using phase of cross-spectral density function. Systems Analysis Modelling Simulation 43, 8, 993-998, (2003)

19. L. Petryka, M. Zych, R. Hanus, J. Sobota, P. Vlasak. Application of the cross-correlation method to determine of solid and liquid velocities during flow in a vertical pipeline. Proc. of 10th ISOPE Ocean Mining \& Gas Hydrates Symp. OMS-2013, Szczecin, Poland, Sept. 22-26, 230-233 (2013) 